\title{
EL ENTORNO FAMILIAR DE CATHALINA DEL SPIRITU SANCTO
}

\section{RAYMOND FAGEL}

Universiteit Leiden r.p.fagel@hum.leidenuniv.nl

RESUMEN: Cathalina del Spiritu Sancto, la autora de la Relación de cómo se ha fundado en Alcántara de Portugal iunto a Lisboa el muy devoto monasterio de N.S. de la Quietación (1627), dedica una parte de su texto a la descripción de su vida personal, lo que da pie al interés por ampliar el conocimiento de la vida de esta religiosa, que se repartió entre los Países Bajos y la Península Ibérica. No se conocen otros textos de su mano. En esta contribución se estudia la información hallada sobre ella y su padre en los Países Bajos y la de sus familiares españoles.

PALABRAS CLAVE: Catalina del Espíritu Santo, Escritoras ibéricas, Portugal, Países Bajos, Historiografía religiosa, Autobiografía, Siglo XVI, Siglo XVII. 


\section{CATHALINA DEL SPIRITU SANCTO'S FAMILY ENVIRONMENT}

ABSTRACT: Cathalina del Spiritu Sancto, the author of the Relación de cómo se ha fundado en Alcántara de Portugal iunto a Lisboa el muy devoto monasterio de N.S. de la Quietación (1627), dedicates part of her text to the description of her personal life, fomenting in this way interest in aumenting our knowledge about the life of this religious author, dividing her life between the Low Countries and the Iberian Peninsula. There are no other known texts by this author. In this contribution, both the documentation on her father and herself in the Low Countries, as well as that regarding her ancestry and her Spanish relatives, are studied.

KEY WORDS: Catalina del Espíritu Santo, Iberian female writers, Portugal, Low Countries, Religious historiography, Autobiography, 16th Century, 17th Century.

$\mathrm{E}^{\mathrm{n}}$ su Relación de cómo se ha fundado en Alcántara de Portugal iunto a Lisboa el muy devoto monasterio de N.S. de la Quietación, la autora, la madre sor Cathalina del Spiritu Sancto, monja del mismo monasterio, aparece descrita en el prólogo por el franciscano Juan de las Llagas de la siguiente manera:

La qual siendo monja professa de Sancta Clara de las urbanas reformadas, biviendo en el monasterio de Hoochstraten, ciu dad de Brabante, fue muy perseguida por ser hija de un cavallero hespañol llamado don Luis Carrillo, governador de aquel condado. (Cathalina del Spiritu Sancto, 1627: prólogo )

Este fraile franciscano de origen flamenco explica que la joven monja fue perseguida en Flandes por un enemigo de su padre, un capitán español de tres compañías de gente a caballo, que en tiempo del Duque de Alba "se avía conjurado contra el Rey de España, y hecho cuerpo con los rebeldes, siguiendo al Príncipe de Orange". El traidor se llamaba don
Alonso López, también apellidado de Herentals por su lugar de residencia. Debido al peligro de la situación, el padre de la monja, en tiempos del Príncipe de Parma, pidió permiso al rey para llevar a su hija a España, lo que terminó en su ingreso en el monasterio de las flamencas en Alcántara (Fagel, 2020). En el capítulo XIII de su relación, la autora vuelve al tema de su vida bajo el título: "De las terribles persecuciones y asaltos que han padecido las religiosas del convento de Hoochstrata, y en especial la authora, siendo moradora en el mismo convento" (Cathalina del Spiritu Sancto, 1627: 26r-31v).

El hecho de que la autora dedique una parte del texto a sucesos autobiográficos, justifica el interés por conocer su vida, dividida entre los Países Bajos y la Península Ibérica. En este breve artículo se tratará en primer lugar de la información hallada sobre padre e hija en los Países Bajos, para finalizar con la descripción de la descendencia y sus familiares españoles. No se pretende ofrecer una visión nueva sobre Cathalina del Spiritu Sancto, sino solamente presentar un contexto histórico para mejor entendimiento de su vida y obra.

\section{Don Luis Carrillo de Castilla}

El padre de Cathalina - no sabemos nada de su madre, lo que podría indicar una relación fuera de matrimonio- era en realidad gobernador del castillo de Hoogstraten desde 1569. En documentos locales se le menciona como don Luis de Castilla (Fernández Bethencourt, 1901: 216). Hoogstraten era un pequeño condado dentro del ducado de Brabante, creado como una entidad aparte gracias a la elevación del famoso cortesano de la época de Carlos V Antonio de Lalaing como su primer conde. Después de la llegada del ejército del Duque de Alba, los bienes de su heredero, Antonio II de Lalaing, fueron confiscados por rebeldía durante la Revuelta en los Países Bajos y como consecuencia don Luis fue nombrado nuevo gobernador del castillo de Hoogstraten, generalmente llamado el 'Gelmelslot'. A pesar de la imagen que transmite la relación de su hija, no era un cargo de gran importancia dentro del gobierno de los Países Bajos. Dos cartas de don Luis, escritas en 1573, son prueba de la 
situación difícil en que se encontró el gobernador durante esos años de conflicto, quedándose con tan sólo ocho soldados, que ni siquiera eran españoles, rodeado de una población en parte hostil:

Oy se son ydos todas las religiosas de Santa Clara a Lira [Lier] y el decano y el cura de Hoochstrate y los canonigos de Hoochstrate tambien se an aubsentado. Yo e visto el mal recaubdo que ay para defender las yglesias y que estos no se quieren declarar enemigos de los enemigos del rey tomando las armas contra ellos, e ordenado de sacar de la yglesia mayor todos los retablos y aornamentos y otras cosas que podrian gastar los hereses, para que se metan en este castillo. An me prometido que lo haran; plega a Dios que sea antes que vengan a robar o a romper lo que ay. ${ }^{1}$

Sus cartas reflejan la imagen de un hombre impotente que intentó salvar la situación dentro de su gobierno, aunque ya había perdido el control del mismo. Medio año después, el Gobernador General de los Países Bajos, Luis de Requesens, escribió lo siguiente en una carta a Felipe II sobre una visita de don Luis a Amberes:

Y aun don Luis Carrillo de Castilla, que ha seis años que es gobernador de Ostrat [Hoogstraten], se me ha venido hoy aqui sin licencia diciendo que los del pueblo le querían matar, y que aquella casa que Vuestra Magestad allí tiene es más casa de placer que castillo, y que él no hizo juramento cuando se lo entregaron, y que no puede guardarle; y cuando los españoles hacen esto, mire Vuestra Magestad, ¿qué harán los de la tierra?²

Obviamente, don Luis había perdido el control de Hoogstraten, saliendo sin permiso de su gobierno.

Don Luis también aparece en la correspondencia del famoso humanista Arias Montano con el igualmente famoso impresor Cristóbal Plantino (Langereis, 2014: 233; Rekers, 1961: 23-30; Dávila Pérez, 2002;

\footnotetext{
${ }^{1}$ Luis Carillo a Juan Moreno, Castillo de Hoogstraten, 4 y 9 de septiembre 1573, Archivo de la Casa de los Duques de Alba, caja 31, 86-87.

${ }^{2}$ Requesens a Felipe II, Amberes, 8 de marzo 1574 (Zabálburu y Sancho Rayón,1892: 314)
}

Domínguez Domínguez, 2017: 182, 190). El humanista español había llegado a Amberes en 1568 para trabajar en la Biblia Políglota, que se preparaba durante esos años en la editorial. Montano era un fiel servidor de Felipe II y del Duque de Alba, pero a la vez mantenía relaciones personales con los humanistas de los Países Bajos, entre ellos también personajes muy afines a las nuevas corrientes religiosas. Alrededor de Plantino existía todo un grupo de humanistas con una mentalidad religiosa muy abierta, que ponían énfasis en la espiritualidad y no en las formas externas de la religión. Plantino llamaba a Arias Montano "su nuevo amigo del alma”.

En todo caso desde 1572, pero quizá ya desde el principio de su estancia en Amberes, el humanista español vivía en una casa de huéspedes regida por una señora llamada Viegas, probablemente de origen portugués, a juzgar por el apellido. Al hacerse en las fuentes referencia también a su hija Ana Herent, parece que se trata de una viuda con una hija medio neerlandesa. La patrona tenía una hermana, que estaba casada nada menos que con Luis Carrillo de Castilla, el gobernador del castillo de Hoogstraten. La correspondencia nos informa de que, cuando la hermana estaba enferma, su marido don Luis iba a menudo visitarla a casa de la señora Viegas, su cuñada. Es más que probable que Arias Montano y don Luis se conocieran en su casa en Amberes. Sabemos con certeza que Montano visitó el castillo de Hoogstraten en búsqueda de libros para la biblioteca de Felipe II en El Escorial. ${ }^{3}$ En una carta de Montano se hace referencia a una conversación con don Luis y el humanista español le ayudó con algún asunto personal.

En 1575-1576, Arias Montano pasó una temporada en Roma. Durante ese período, Plantino le escribió sobre su visita a la casa de la señora Viegas, comentándole que esperaba poder hacerlo muchas más veces. Era fácil, porque la casa se encontraba en su camino habitual hacia su huerta. La hermana permanecía enferma en la casa y Plantino también había hablado con ella. En otra carta, Plantino informaba a Monta-

${ }^{3}$ Montano al secretario Zayas, Amberes, 28 de febrero 1569, 6 de Abril 1569 y 7 de enero 1570, Colección de documentos inéditos (1862: 149-150, 153-154, 167).

UNED. REI, 8 (2020), pp. 41-52

ISSN 2340-9029 
no de que realmente visitaba la casa a menudo, siempre con la hermana enferma presente. Durante estas visitas, Plantino intentaba siempre dejar cartas de Montano en secreto a la hija de la señora Viegas, que mantenía una relación espiritual con el humanista. Plantino describe cómo a menudo había incluso una cuarta mujer en la casa, porque la hermana enferma, la mujer de Luis Carrillo, recibía frecuentemente visitas de "la hija del mismo capitán”. En esas ocasiones, todos los visitantes conincidían en la misma habitación. Esta hija muy probablemente sea Cathalina, la autora de la relación, antes de entrar en el convento de las clarisas en Hoogstraten.

Es decir, creemos que Cathalina conoció personalmente al gran editor Plantino y es más que probable que también conociera a Arias Montano. En todo caso, su pariente, la sobrina de la mujer de su padre, mantenía una estrecha amistad con el humanista español. Algo de la espiritualidad de estas personas debe haber sentido en sus conversaciones. Y quizá Cathalina tuvo un vínculo con Portugal a través de la familia Viegas antes de llegar a ese país. ${ }^{4}$ Cabe recordar asimismo que el editor de su Relación, Pedro Craesbeeck, antes de su mudanza a Lisboa, había trabajado en la editorial de Plantino en Amberes, y es posible que la joven Cathalina también hubiera conocido más figuras del entorno de Plantino y Montano.

\section{El árbol genealógico de Cathalina}

En 1577, un don Luis Carrillo de Acuña estaba presente en el castillo de Amberes cuando su gobernador, el famoso héroe militar Sancho Dávila, tuvo que entregar el castillo al Duque de Aarschot (Dávila y San Vítores, 1713: 212, 215). El acuerdo entre el Rey y sus súbditos neerlandeses de este año incluía que todos los castillos y fortalezas deberían estar en manos de gente del propio país. Sin duda alguna, también don Luis se vio forzado de dejar su gobierno de Hoogstraten, pero a diferencia de

${ }^{4}$ Plantino a Montano, Amberes, 29 de octubre 1575 y 13-16 de enero 1576 (Dávila Pérez, 2002: 263, 321)
Sancho Dávila, se puede creer que el padre de Cathalina dejó su puesto sin ninguna objeción por su parte.

Partiendo de la idea de que este don Luis Carrillo de Acuña fuese la misma persona que el llamado don Luis Carrillo de Castilla, fue posible encontrar a la familia de don Luis, y de Cathalina. Se trata de la familia de los señores de Pinto. Gómez Carrillo de Acuña (1400-1441), señor de Jadraque, se casó en 1434 con María de Castilla y Salazar, nieta del rey Pedro I, el Cruel, de Castilla. Su padre, Diego de Castilla, era hijo natural del rey (Valdaliso Casanova, 2018), mientras que Gómez Carrillo de Acuña era el hijo de un noble portugués. De este matrimonio nació Alonso Carrillo de Acuña, que se casó con Leonor de Toledo, la heredera del señorío de Pinto. Posteriormente, Alonso obtuvo también el señorío de Caracena (Quijorna Rodríguez, 2012: 218).

El hijo de Alonso, Gómez Carrillo de Acuña y Castilla, era el sexto señor de Pinto, pero también se le conoce como Gómez Carrillo de Toledo (Fernández de Oviedo, 1880: batalla I, quinquagena 4, diálogo 43; Fernández Bethencourt, 1901: 214-216; Quijorna Rodríguez, 2012: 223). En su testamento del 17 de marzo de 1530, Gómez expresaba su voluntad de ser enterrado en la capilla central del monasterio de los franciscanos en Pinto, vestido de franciscano. De su segundo matrimonio con Beatriz Sarmiento, hija del Conde de Salinas, nació Alonso Carrillo de Toledo (o de Acuña), mientras que de su tercer matrimonio con María Manrique de Ayala nacieron dos hijos, Gómez Carrillo de Castilla y Pedro Xuárez de Castilla, y una hija, Juana de Castilla. Pero Gómez también tuvo un hijo natural, y este es nuestro don Luis Carrillo de Castilla, el gobernador del castillo de Hoogstraten y el padre de Cathalina.

Es decir, Cathalina fue, con gran probabilidad, la hija natural del hijo natural de un miembro de una familia que descendía de una rama bastarda de los reyes de Castilla. Los descendientes del rey Pedro de Castilla incluso forjaron un "sentimiento de pertenencia a un mismo linaje" (Valdaliso Casanova, 2018). A través de su padre y de sus medio hermanos, don Luis estaba relacionado con algunas familias de la alta nobleza. Su medio hermano Gómez Carrillo murió sin descendientes y de Pedro Xuárez sabemos que estuvo involucrado como capitán en el rapto de Feli- 
pe Guillermo de Orange, el hijo mayor de Guillermo de Orange, cuando le llevaron en dirección de España como rehén en 1568. Posteriormente, Pedro Xuárez actuaría como inspector de las fortalezas en el reino de Nápoles.

A la única hija de Gómez Carrillo, el sexto señor de Pinto, se la conoce como Juana de Castilla. Por matrimonio concertado se convirtió alrededor de 1529 (a la edad de 14 años) en la esposa de Hernando Niño, un toledano de grandes riquezas y de gran influencia. Su marido falleció en 1562, sin que la pareja hubiera tenido hijos. Después de su muerte, Juana empezó a concentrar sus esfuerzos en la fundación de un monasterio. En principio su objetivo era fundar un monasterio de carmelitas descalzas o de monjas agustinas, pero finalmente se decidió por un convento de pobres dominicanas en Toledo, llamado Jesús y María. La fundación se materializó en 1599. Esta señora de Los Tejares murió el 27 de agosto de 1619 en Toledo, cuando ya había pasado los cien años de edad (Martz, 2003: 108; Franco Silva, 2001; Canabal Rodríguez, 2006; Canabal Rodríguez, 2018).

Los hijos del segundo matrimonio de Gómez Carrillo de Acuña y Castilla continuaron la línea de los señores de Pinto. Su hijo Alonso Carrillo de Toledo, nacido alrededor de 1483, se convirtió en el séptimo señor de Pinto, seguido por su hijo Luis Carrillo de Toledo, octavo señor de Pinto, y el hijo de este, igualmente llamado Luis Carrillo de Toledo, quien se convirtió en el primer marqués de Caracena y el primer conde de Pinto (Rama Patiño y Vázquez Lijó, 2018; Quijorna Rodríguez, 2012: 225). Este personaje de la alta nobleza, a través de tres matrimonios, bien relacionado con otras familias de gran influencia, llegó a ser gobernador de Galicia y virrey de Valencia. El primer marqués (1564-1626) fue sin duda alguna el familiar más influyente en la corte durante la última parte de la vida de Cathalina. Tanto el marqués como su mujer mantenían contacto con personajes del mundo religioso tan ilustres como la poeta y activista religiosa Luisa de Carvajal y Mendoza, quien en una carta a

${ }^{5}$ En 1574 murió en la batalla de Reimerswaal un capitán llamado Carrillo Acuña (Barrado y Font, 1906: 88)

UNED. REI, 8 (2020), pp. 41-52

ISSN $2340-9029$
Magdalena de San Jerónimo demostró de manera concisa la importancia de la Revuelta en los Países Bajos para España: "Nos toca a todos en España, todo cuanto a Flandes toca" (Rodríguez Pérez, 2008: 12; Cruz, 2014). ${ }^{6}$ Es decir, estos familiares de Cathalina tenían un gran interés en la espiritualidad de la época y conocían perfectamente los problemas de los católicos en los Países Bajos. En los documentos y cartas que nos han dejado estos marqueses de Caracena es donde existe la posibilidad de hallar en el futuro pruebas de sus posibles contactos con Cathalina o al menos del conocimiento de su existencia (Franco Silva, 1996: 231). ${ }^{7}$

El nieto del primer marqués, Luis Francisco de Benavides y Carrillo de Toledo, alcanzaría el cargo de Gobernador General de los Países Bajos (1659-1664), gobernando los territorios donde don Luis y su hija Cathalina habían vivido casi un siglo antes. En 1665, al final de la guerra de restauración en Portugal, intentó conquistar Lisboa, pero acabó perdiendo la famosa batalla de Montes Claros, llevándole así también al otro territorio donde había vivido Cathalina (Herrero Sánchez, 2018).

Aunque es difícil averiguar si Cathalina mantuvo contactos con sus familiares, podemos afirmar que se trataba de personas de la alta nobleza, bien conectadas con la corte del rey y con la iglesia católica. Su abuelo Gómez ya se enterró en hábito de franciscano en el monasterio de Pinto, su tía Juana era una de las mujeres más ricas de Toledo y fundadora de un convento, y el apellido de Castilla dejaba ver que la familia descendía de los reyes de Castilla. En este sentido Cathalina y el rey Felipe II estaban emparentados, aunque es discutible que el monarca fuera consciente de la existencia de tal relación.

La información acerca de su padre y el círculo de Amberes refleja un ambiente completamente diferente: el de Plantino, Arias Montano y los comandantes militares españoles en Flandes. Sobre ello disponemos de documentación que afirma que Cathalina del Spiritu Sancto muy probablemente conoció personalmente al editor más famoso de los Países

${ }^{6}$ Carvajal y Mendoza (1965). La carta a Magdalena de San Jerónimo es del 10 de septiembre de 1601

Archivo Histórico de la Nobleza, Ducado de Frías, Archivo Marquesado de Caracena.

UNED. REI, 8 (2020), pp. 41-52

ISSN 2340-9029 
Bajos y posiblemente también al humanista y librero de El Escorial. También que vivió la primera parte de la Revuelta de los Países Bajos muy de cerca, siendo perseguida por un capitán español al servicio del príncipe de Orange. La autora de la Relación de cómo se ha fundado en Alcántara de Portugal iunto a Lisboa el muy devoto monasterio de N.S. de la Quietación, no fue una monja sin conocimiento del mundo fuera del convento.

Recibido: $18 / 03 / 2020$

Aceptado: 19/09/2020

\section{Referencias citadas}

Barrado y Font, Francisco (1906), D. Luis de Requesens y la política española en los Países Bajos, Madrid: Real Academia de la Historia.

Canabal Rodríguez, Laura (2006), "Clausura en el siglo XVII: el convento dominicano de Jesús y María”, Toletum. Cuestiones de Teología e Historia, 7, pp. 137-160.

Canabal Rodríguez, Laura (2018), “Juana de Castilla y Toledo”, Real Academia de la Historia, Diccionario biográfico electrónico <http://dbe.rah. es/> [consultado 1 de Agosto, 2019].

Carvajal y Mendoza, Luisa (1965), Epistolario y poesías, ed. J. González Marañón, Jesús y C. M. Abad, Madrid: Atlas.

Cathalina del Spiritu Sancto (1627), Relacion de como se ha fundado en Alcantara de Portugal iunto a Lisboa el muy devoto monasterio de N.S. de la Quietación..., Lisboa: Pedro Craesbeeck.

Colección de documentos inéditos para la historia de España (1862), vol. XLI, Madrid: Real Academia de la Historia.

Cruz, Anne J. (2014), The Life and Writings of Luisa de Carvajal y Mendoza, Toronto: ACMRS Press.
Dávila Pérez, Antonio, ed. (2002), Benito Arias Montano. Correspondencia conservada en el Museo Plantin-Moretus de Amberes, Alcañiz/ Madrid: Instituto de Estudios Humanísticos "Palmyrenus”, 2 vols.

Dávila y San Vítores, Gerónimo Manuel (1713), El rayo de la guerra, hechos de Sancho Dávila, Valladolid: Antonio de Figueroa.

Domínguez Domínguez, Juan Francisco, ed. (2017), Benito Arias Montano. Correspondencia, vol. I (1560-1570), Madrid: Ediciones Clásicas.

Fagel, Raymond (2020), “Orange's Spanish mulatto and other side-changers. Narratives on Spanish defection during the Revolt in the Low Countries", Raymond Fagel, Leonor Álvarez Francés y Beatriz Santiago Belmonte (eds.), War narratives and the Revolt in the Low Countries, Manchester: Manchester University Press, pp. 107-124.

Fernández Bethencourt, Francisco (1901), Historia genealógica y heráldica de la monarquía española, vol. III, Madrid: Jaime Ratés.

Fernández de Oviedo, Gonzalo (1880), Batallas y quinquagenas, Vicente de la Fuente ed., Madrid: Real Academia de la Historia.

Franco Silva, Alfonso (1996), La fortuna y el poder. Estudios sobre las bases económicas de la aristocracia castellana (s. XIV-XV), Cádiz: Universidad de Cádiz.

Franco Silva, Alfonso (2001), "Los Niño. Un linaje de la oligarquía municipal de Toledo en el siglo XVI", Anuario de Estudios Medievales, 31, pp. 191-294.

Herrero Sánchez, Manuel (2018), "Luis de Benavides Carrillo de Toledo", Diccionario biográfico electrónico <http://dbe.rah.es/> [consultado 23 de Septiembre, 2020].

Langereis, Sandra (2014), De woordenaar. Christoffel Plantijn. 's Werelds grootste drukker en uitgever 1520-1589, Amsterdam: Uitgeverij Balans.

Martz, Linda (2003), A Network of Converso Families in Early Modern Toledo, Ann Arbor: University of Michigan Press. 
Quijorna Rodríguez, Ana (2012), "Mecanismos y estrategias de promoción y consolidación de los Carrillo de Toledo, señores de Caracena y Pinto", Historia y genealogía, 2, pp. 215-237.

Rama Patiño, Luz, y José Manuel Vázquez Lijó (2018), "Luis Carrillo de Toledo", Diccionario biográfico electrónico <http://dbe.rah.es/> [consultado 23 de Septiembre, 2020].

Rekers, Bernard (1961), Benito Arias Montano 1527-1598. Studies over een groep spiritualistische humanisten in Spanje en de Nederlanden, op grond van hun briefwisseling, Groningen: V.R.B..

Rodríguez Pérez, Yolanda (2008), The Dutch Revolt through Spanish Eyes. Self and Other in Historical and Literary Texts of Golden Age Spain (ca. 1548-1673), Bern y Oxford: Peter Lang.

Valdaliso Casanova, Covadonga (2018), "Diego de Castilla y de Sandoval", Real Academia de la Historia, Diccionario biográfico electrónico $<$ http://dbe.rah.es/> [consultado 23 de Septiembre, 2020].

Zabálburu, Francisco de y José Sancho Rayón, eds. (1892), Nueva colección de documentos inéditos para la historia de España y de sus Indias, tomo I, Madrid: M.G. Hernández. 